\title{
Nature of Conduction in Doped Silicon
}

\author{
Tae-In Jeon and D. Grischkowsky \\ School of Electrical and Computer Engineering and Center for Laser and Photonics Research, \\ Oklahoma State University, Stillwater, Oklahoma 74078
}

(Received 1 July 1996)

\begin{abstract}
Via ultrafast optoelectronic $\mathrm{THz}$ techniques, we are able to test alternative theories of conduction by precisely measuring the complex conductivity of doped silicon from low frequencies to frequencies higher than the plasma frequency and the carrier damping rate. These results, obtained for both $n$ and $p$-type samples, spanning a range of more than 2 orders of magnitude in the carrier density, do not fit any standard theory. We only find agreement over the full frequency range with the complex conductivity given by a Cole-Davidson type distribution applied here for the first time to a crystalline semiconductor, and thereby demonstrate that fractal conductivity is not just found in disordered material.
\end{abstract} [S0031-9007(96)02284-3]

PACS numbers: $72.30 .+\mathrm{q}$

The frequency dependent complex conductivity is one of the most basic properties describing doped semiconductors. Associated with the conductivity are the key parameters characterizing the dynamics of the free carriers in semiconductors, the plasma frequency $\omega_{\mathrm{p}}$, and the carrier damping rate $\Gamma=1 / \tau$, where $\tau$ is the carrier collision time. Characteristically, $\omega_{\mathrm{p}}$ and $\Gamma$ have THz frequencies. Even though the complex conductivity has been a topic of theoretical studies for several decades, complete experimental characterizations from low frequencies to beyond several $\mathrm{THz}$ have only started to be performed $[1,2]$.

Here we report definitive measurements of the complex conductivity from de to $2.5 \mathrm{THz}$ on doped silicon. Compared to earlier experimental studies of doped silicon $[1,3]$, these new results have sufficient frequency range and precision to test alternative theories [4-12] for the conductivity. However, for both $n$ - and $p$-type silicon and over a measured range of more than 2 orders of magnitude of the carrier density we do not find agreement with any standard theory, including Drude, lattice-scattering, and impurity-scattering theories. As a result, we were forced to look outside the standard theoretical approaches.

Our interest in a Cole-Davidson type distribution was motivated by the close relationship between the Debye theory of dielectric insulators and Drude theory, the simplest theory of electrical conduction. For Debye theory, in response to a step-function electric field, the polarization is established exponentially with a characteristic response time. Similarly, for Drude theory, in response to a stepfunction $E$ field, the current is established exponentially with the carrier collision time. Consequently, in the frequency domain the mathematical representations of these two theories are identical. It has been found experimentally that for relatively low frequencies the complex dielectric constants of disordered materials, such as molecular liquids [13], polymers [14], and more recently ionic glasses $[15,16]$, show better agreement with a modified Debye spectral response, known as the Cole-Davidson (C-D) distribution. In the frequency domain, the C-D distribution corresponds to Debye theory with a fractional exponent $\beta$, limited to values between 0 and 1 , and reduces to Debye theory for $\beta=1$.

In this paper, for the first time, we have modified the frequency dependent complex conductivity of Drude theory to include the C-D fractional exponent $\beta$, and thereby, achieve an exceptional fit to all of our measurements. This first application of a C-D type distribution to fit the complex conductivity of well-ordered crystalline semiconductors extends the demonstrated frequency range of this type distribution by more than 3 orders of magnitude and demonstrates that fractal conductivity is not just found in disordered material. Niklasson [16] has stated that a C-D type distribution can be obtained from a fractal conduction process with a cutoff, and relating this to percolation theory would predict $\beta=0.72$. Our measured $\beta$ values range from 0.73 to 1 , where $\beta$ approaches unity (Drude theory) as the number density of carriers is increased. For approximately the same dc conductivity, $\beta$ is closer to unity for $n$-type than $p$-type silicon.

In order to better understand these frequency-domain results, we compare the corresponding Drude and C-D time-domain responses. The time-domain response function of Drude theory is the single sided exponential $\exp (-t / \tau)$ [17]. However, for the C-D case the response function has a much faster initial decay which asymptotically approaches the Drude response [15]. This behavior shows that the C-D response function has a distribution of relaxation times; this distribution is known analytically [15] and appears to be a more realistic representation of our measurements. For a step function electrical pulse, the intuitively satisfying, initial Drude response is the ballistic acceleration $e E / m^{*}$ of the carriers, $e$ is the carrier charge, $E$ is the applied field, and $m^{*}$ is the effective mass. The corresponding C-D response to a step-function $E$ field is initially much faster than the Drude response, and more rapidly approaches the same asymptotic dc current as for Drude, thereby indicating a smaller high frequency effective mass than the low frequency value. 
The THz time-domain spectroscopy (THz-TDS) measurements were performed by optoelectronically measuring the amplitude of freely propagating subpsec $\mathrm{THz}$ electromagnetic pulses transmitted through the silicon wafer under investigation [1,2,18]. These transmitted pulses were then compared to the measured $\mathrm{THz}$ pulses with no sample in place. The $\mathrm{THz}$ system was in an enclosure maintained at a relative humidity of less than $2 \%$ to mitigate the effects of water vapor. Analysis of the respective complex numerical Fourier transforms determines the frequency dependent absorption and index of refraction [18]. The 51-mm-diam silicon wafers, obtained from Virginia Semiconductor Inc., had $\langle 100\rangle$ orientation, were doped with phosphorus ( $n$-type) or boron ( $p$-type), and had thicknesses ranging from $5 \mathrm{~mm}$ to $50 \mu \mathrm{m}$, depending on carrier density.

The frequency dependent complex dielectric constant $\varepsilon$ is equal to the square of the complex index of refraction $n=n_{r}+i k$. The imaginary index $k$ is determined by measuring the power absorption coefficient $\alpha=k 4 \pi / \lambda_{0}$, where $\lambda_{0}$ is the free-space wavelength. The dielectric response for doped silicon is described by the following general relationship:

$$
\varepsilon=\varepsilon_{\mathrm{Si}}+i \sigma /\left(\omega \varepsilon_{0}\right),
$$

where $\varepsilon_{\mathrm{Si}}$ is the dielectric constant of undoped $\mathrm{Si}, \sigma$ is the complex conductivity, and $\varepsilon_{0}$ is the free-space permittivity. The index of refraction $n_{r}=3.417$ and absorption coefficient $\alpha \sim 0$ have been measured [18] for undoped silicon over the frequency range considered here.

The theoretical approaches discussed in this paper are described by the equations

$$
\begin{aligned}
& \sigma=\varepsilon_{0} \omega_{\mathrm{p}}^{2} \tau /(1-i \omega \tau)^{\beta}, \\
& \sigma=i \varepsilon_{0} \omega_{\mathrm{p}}^{2}\langle 1 /[\omega+i \Gamma(E)]\rangle,
\end{aligned}
$$

where the plasma frequency $\omega_{\mathrm{p}}$ is given by $\omega_{\mathrm{p}}^{2}=N e^{2} /$ $\left(\varepsilon_{0} m^{*}\right) ; m^{*}=0.26 m_{0}$ for the effective electron mass and $m^{*}=0.37 m_{0}$ for the effective hole mass; $m_{0}$ is the freeelectron mass.

For Drude theory $\beta=1$ in Eq. (2a). The ColeDavidson distribution used here is given by Eq. (2a), which appears as the complex conjugate of the C-D distribution from Debye theory [13-16], due to the phase quadrature relation between conductivity and dielectric polarization. What we will call "scattering theory" [1] is given by Eq. (2b) with $\Gamma(E)=\Gamma_{l}\left(E / k_{B} T_{0}\right)^{1 / 2}+$ $\Gamma_{i}\left(E / k_{B} T_{0}\right)^{-3 / 2}$ describing the damping rate of carriers with energy $E$ relative to the bottom of the conduction band; $\Gamma_{l}$ and $\Gamma_{i}$ are coupling constants characterizing the efficiency of scattering with the lattice and the ionized impurities, respectively, and depend on temperature and doping; $k_{B}$ is the Boltzmann constant; $T_{0}$ is a normalization parameter with the dimension of temperature. The symbols \langle\rangle denote an average, weighted by the product of the distribution of the carriers multiplied by their energy. For the parabolic band considered here the weight factor is $E^{3 / 2} \exp -\left(E / k_{B} T\right)$. For all the theories the $\mathrm{dc}$ conductivity is given by $\sigma_{\mathrm{dc}}=e \mu N$, with the mobil- ity $\mu=e /\left(\Gamma m^{*}\right)$ for Drude and Cole-Davidson, and $\mu=\left(e / m^{*}\right)\langle 1 / \Gamma(E)\rangle$ for scattering theory. We have numerically verified that Eqs. (2a) and (2b) satisfy the Kramers-Kronig relations.

For all our samples we find agreement with Drude theory [5] for the lowest frequencies. However, as the frequency increases beyond the carrier damping rate $\Gamma / 2 \pi$, the measured real part of the conductivity becomes significantly larger than the simple Lorentzian shape of Drude theory, while the measured peak of the imaginary part, occurring approximately at $\Gamma / 2 \pi$, is significantly lower than the Drude result. These deviations are strongest for

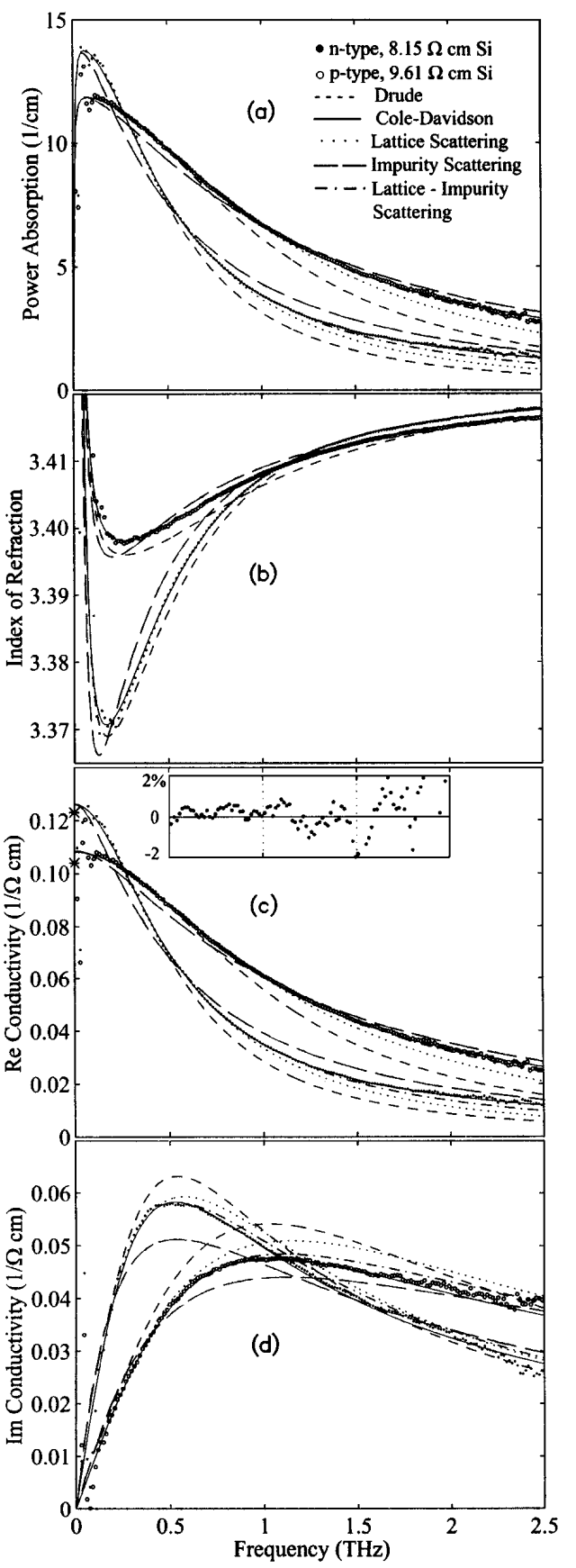

FIG. 1. Measurements and theoretical comparisons; see text. 
the lightest doping and decrease as the doping level is increased.

If our results are fit with the more complete scattering theory [1], where the carrier relaxation is due to both electron-phonon (lattice) collisions and carrier scattering from ionized impurities, the comparison is not selfconsistent. At the lowest number densities of carriers $N$, where our results should be fit with only lattice collisions, the results are best fit with impurity scattering as the major process and lattice scattering as the minor process. In contrast, at much higher $N$ the relative proportion of lattice scattering must be increased (rather than decreased) to obtain reasonable agreement with the measurements.

Our THz-TDS measurements of the THz power absorption coefficient of our highest resistivity, 5-mm-thick, Si samples shown in Fig. 1(a) are the averages of nine independent measurements. Typically, the ratios of individual absorption measurements vary from unity by $\pm 1 \%$ over the frequency band from 0.1 to $1.5 \mathrm{THz}$, while the ratios of the index of refraction vary by $\pm 0.02 \%$ from 0.1 to $2.5 \mathrm{THz}$. Because of the frequency response of the THzTDS system, the data is reliable only above $0.08 \mathrm{THz}$. We also show in Fig. 1(a) an earlier absorption measurement [3] (squares) of nonintentionally doped $9.4 \Omega \mathrm{cm}$, $n$-type silicon made with a synchrotron radiation source and bolometric power detection.

For our entire series of absorption measurements the experimental results are bracketed between the predictions of Drude theory and those of scattering theory with only impurity scattering. Only the C-D distribution can fit the measurements over the entire frequency range. The fit in Fig. 1(a) is so precise that only at the lowest and highest frequencies can the C-D fitting line be seen separate from the data. The fitting parameters are given in Table I, together with the corresponding number densities and mobilities.
The measured indices of refraction in Fig. 1(b) show strong frequency dependences and well resolved minima that are only fit by the C-D distribution. Although the results of Figs. 1(a) and 1(b) are related by the KramersKronig relationships, they are measured independently. The ability to simultaneously obtain good fits with the same parameters for both the absorption and index of refraction shows the accuracy of THz-TDS to measure the complex transmitted spectrum.

Given these measurements of the absorption and index of refraction, Eq. (1) determines the real $\sigma_{r}$ and the imaginary $\sigma_{i}$ parts of the conductivity shown in Figs. 1(c) and 1(d). In Fig. 1(c) the experimental results are only well fit by the C-D distribution, whose fitting line almost vanishes into the presented data. The inset shows the deviation between the measurements and the C-D distribution to be less than $1 \%$ from 0.5 to $1.5 \mathrm{THz}$. At zero frequency the 4-point probe measurements [values are stated in Fig. 1(a)] are indicated by the asterisks. Because all the fits originate from the same value at $\omega=0$, the product $\mu N$ is the same for all the theories. The calculated C-D distribution also fits well to the measured imaginary part of the conductivity which differs significantly with that predicted by Drude and scattering theory.

Our corresponding THz-TDS measurements of the much lower resistivity $200-\mu$ m-thick, $S i$ wafers are shown in Fig. 2. The $p$-type measurements have a much lower signal-to-noise ratio due to excessive absorption, because the wafer was too thick. However, the measurements can still discriminate between the various theoretical predictions. The measured conductivities are approximately 50 times larger than those shown in Fig. 1. This situation broadens the frequency response due to the 7 and 10 times increase in $\omega_{\mathrm{p}}$ for the $n$ - and $p$-type $\mathrm{Si}$, respectively, together with the 1.5 and 1.9 times increase in $\Gamma$ for the

TABLE I. Theoretical fitting parameters ( $p$-type in parentheses) for the measurements presented in Figs. 1 and 2 . Frequencies are in THz; mobilities are in $\mathrm{cm}^{2} / \mathrm{V} \mathrm{sec}$; multiply $N$ by $10^{14} \mathrm{~cm}^{-3}$ for the Fig. 1 entry; multiply $N$ by $10^{16} \mathrm{~cm}^{-3}$ for the Fig. 2 entry.

\begin{tabular}{|c|c|c|c|c|c|}
\hline & Drude & C-D & Lattice & Impurity & Scattering \\
\hline \multicolumn{6}{|l|}{ Fig. 1} \\
\hline$\omega_{\mathrm{p}} / 2 \pi$ & $0.350(0.448)$ & $0.323(0.404)$ & $0.37(0.49)$ & $0.44(0.59)$ & $0.393(0.540)$ \\
\hline$\Gamma / 2 \pi$ & $0.54(1.03)$ & $0.46(0.84)$ & $x$ & $x$ & $x$ \\
\hline$\beta$ & $x$ & $0.84(0.76)$ & $x$ & $x$ & $x$ \\
\hline$\Gamma_{i} / 2 \pi$ & $x$ & $x$ & 0 & $3.8(8.0)$ & $1.695(4.690)$ \\
\hline$\Gamma_{l} / 2 \pi$ & $x$ & $x$ & $0.45(0.90)$ & 0 & $0.105(0.138)$ \\
\hline$N$ & $4.0(9.2)$ & $3.4(7.4)$ & $x$ & $x$ & $5.0(13.0)$ \\
\hline$\mu$ & $2000(730)$ & $2340(900)$ & $x$ & $x$ & $1580(490)$ \\
\hline \multicolumn{6}{|l|}{ Fig. 2} \\
\hline$\omega_{\mathrm{p}} / 2 \pi$ & $2.653(4.490)$ & $2.53(4.28)$ & $2.75(4.82)$ & $3.20(5.91)$ & $2.863(5.320)$ \\
\hline$\Gamma / 2 \pi$ & $0.838(1.985)$ & $0.76(1.80)$ & $x$ & $x$ & $x$ \\
\hline$\beta$ & $x$ & $0.91(0.86)$ & $x$ & $x$ & $x$ \\
\hline$\Gamma_{i} / 2 \pi$ & $\times$ & $x$ & 0 & $5.5(15.5)$ & $1.98(8.20)$ \\
\hline$\Gamma_{l} / 2 \pi$ & $\times$ & $\times$ & $0.67(1.70)$ & 0 & $0.2(0.3)$ \\
\hline$N$ & $2.3(9.3)$ & $2.1(8.4)$ & $x$ & $x$ & $2.6(13.0)$ \\
\hline$\mu$ & $1280(380)$ & $1420(420)$ & $x$ & $x$ & $1110(260)$ \\
\hline
\end{tabular}




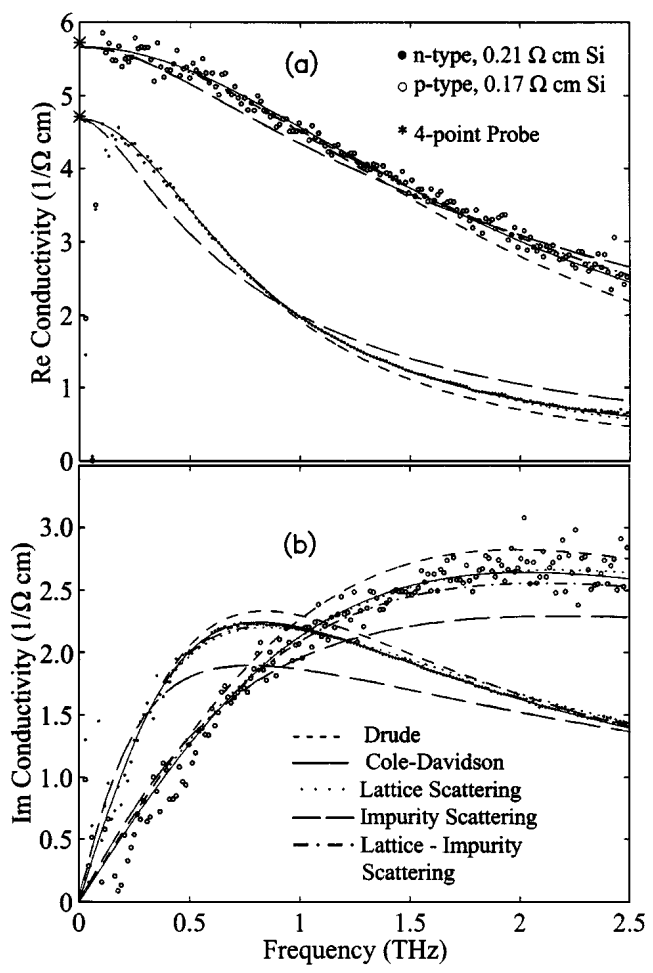

FIG. 2. Measurements and theoretical comparisons; see text.

$n$ - and $p$-type, respectively, as presented in Table I. For the $n$-type material we obtain agreement with the real and imaginary parts of the conductivity only with the C-D distribution and with $\beta=0.91$. This test of the distribution is not as severe as in Fig. 1, because the frequency extent is not as large compared to $\Gamma / 2 \pi$. This comment is especially true for the $p$-type results for which $\Gamma / 2 \pi$ is of the same order as the measured frequency range.

Figure 3 summarizes the entire series of measurements, by presenting the mobilities, carrier densities, and C-D parameters $\beta$ for the best fits to experiment. The measured mobilities monotonically decrease with increasing carrier density, thereby confirming that the main scattering impurities are the $n$ - or $p$-type dopant. Thus, the contradictory behavior with respect to the relative proportion of lattice or impurity scattering cannot be explained by unknown impurity effects. The dependence of the mobility on carrier density for the three theoretical fits to the data are all fit by the empirical Caughey-Thomas curve [19] with different parameters. At the lower carrier densities the C-D and Drude mobilities are much higher than previous measurements [3,19]. At carrier densities above $10^{17} \mathrm{~cm}^{-3}$ these differences disappear and the theories converge. For the $n$-type samples the C-D parameter $\beta$ increases with number density. For the two highest number densities $\beta=1$, and C-D theory has reduced to Drude theory. For the $p$-type samples $\beta$ shows a more abrupt increase as the number density is increased.

We acknowledge stimulating and informative discussions with R. A. Cheville, R. W. McGowan, and Xincheng

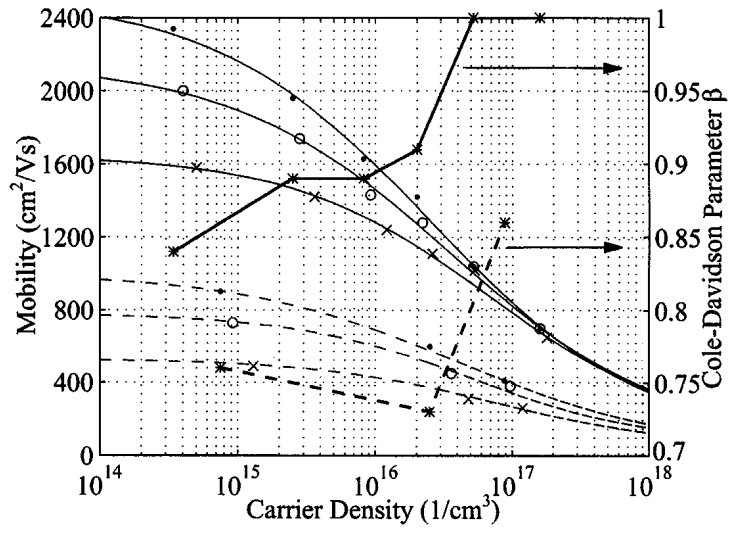

FIG. 3. Mobilities, carrier number densities, and C-D parameters for the Drude $(\bigcirc)$, Cole-Davidson $(\bullet)$, and scattering $(\times)$ theory fits to our measurement series on six $n$-type (solid lines) and three $p$-type (dashed lines) Si wafers. The C-D parameters are joined by the straight lines ( $n$-type, solid line; $p$-type, dashed line).

Xie. This work was partially supported by the National Science Foundation under Grant No. PHY-9422952.

[1] M. van Exter and D. Grischkowsky, Appl. Phys. Lett. 56, 1694 (1990); Phys. Rev. B 41, 12140 (1990).

[2] N. Katzenellenbogen and D. Grischkowsky, Appl. Phys. Lett. 61, 840 (1992).

[3] T. Ohba and S.-I. Ikawa, J. Appl. Phys. 64, 4141 (1988).

[4] M. Vindevoghel, J. Vindevoghel, and Y. Leroy, Infrared Phys. 15, 161 (1975).

[5] N.W. Ashcroft and N.D. Mermin, Solid State Physics (Holt, Rinehart, and Winston, New York, 1976).

[6] C. Herring and E. Vogt, Phys. Rev. 101, 944 (1956).

[7] D. Long and J. Myers, Phys. Rev. 120, 39 (1960).

[8] D. Long, Phys. Rev. 120, 2024 (1960).

[9] J. M. Ziman, Electrons and Phonons (Oxford University Press, London, 1960).

[10] P. A. Schumann, Jr. and R. P. Phillips, Solid State Electron. 10, 943 (1967).

[11] P. K. Basu and B. R. Nag, Phys. Status Solidi B 53, K61 (1972).

[12] E. Barta, Infrared Phys. 17, 111 (1977).

[13] D. W. Davidson and R. H. Cole, J. Chem. Phys. 19, 1484 (1951).

[14] S. Havriliak, Jr. and D. G. Watts, Polymer 27, 1509 (1986).

[15] K. Pathmanathan and J. R. Stevens, J. Appl. Phys. 68, 5128 (1990).

[16] G. A. Niklasson, K. Brantervik, and L. Borjesson, J. NonCryst. Solids 131, 1096 (1991).

[17] D. Grischkowsky and N. Katzenellenbogen, in OSA Proceedings on Picosecond Electronics and Optoelectronics, Salt Lake City, Utah, 1991, edited by T. C. L. G. Sollner and J. Shah (Optical Society of America, Washington, DC, 1991), Vol. 9.

[18] D. Grischkowsky, S. Keiding, M. van Exter, and Ch. Fattinger, J. Opt. Soc. Am. B 7, 2006 (1990).

[19] D. M. Caughey and R.F. Thomas, Proc. IEEE 50, 2192 (1967). 